\title{
AN EMBEDDING-OBSTRUCTION FOR PROJECTIVE VARIETIES ${ }^{1}$
}

\author{
BY AUDUN HOLME
}

Communicated by A. Mattuck, January 29, 1974

A classical problem in differential topology is the following: Let $X$ be a compact $n$-dimensional differentiable manifold (without boundary). Then compute the least integer $m=m(X)$ such that $X$ may be embedded into $\boldsymbol{R}^{m}$. Usually this question is attacked as follows (see Atiyah [1]): (a) An upper bound for $m$ is obtained by exhibiting explicit embeddings, and (b) a lower bound is obtained by certain homotopy invariants.

The forthcoming paper [2] deals with an algebro-geometric counterpart to the problem mentioned above: Let $X$ be a nonsingular, projective $k$-variety embedded in some projective space $\boldsymbol{P}_{k}^{N}$ by the embedding $i$. For simplicity we assume the field $k$ to be algebraically closed, but the results of [2] still hold under the weaker assumption that $k$ is infinite. The main result is that the least integer $m=m(X, i)$, such that $X$ can be embedded into $\boldsymbol{P}_{k}^{m}$ via a projection from $\boldsymbol{P}_{k}^{N}$, is effectively computed in terms of the degrees of the Chern-classes of $X$.

More precisely, let $X \subseteq \boldsymbol{P}_{k}^{N}$ be an $n$-dimensional nonsingular projective variety, embedded in $\boldsymbol{P}_{k}^{N}$. Let $c_{i}=c_{i}(X)=c_{i}\left(\Omega_{X / k}^{1}\right) \in A(X)$ be the Chernclasses of $X$, where $A(X)$ denotes the Chow-ring of $X$. Consider the formal inverse of the alternating Chern-polynomial:

$$
\left[\sum_{i=0}^{n}(-1)^{i} c_{i} T^{i}\right]^{-1}=\sum_{i=0}^{\infty} f_{i} T^{i}
$$

Here $f_{i}=0$ for $i>n$. Let $d_{i}=\operatorname{deg}\left(f_{i}\right)$ with respect to the embedding $i: X \varsigma$ $\boldsymbol{P}_{k}^{N}$. In particular $d_{0}=\operatorname{deg}(i(X))=d$. Define

$$
B_{X}(T)=\left(\sum_{i=0}^{n} d_{i} T^{i}\right)\left(\sum_{i=0}^{2 n+1}\left(\begin{array}{c}
2 n+2 \\
i
\end{array}\right) T^{i}\right)=B_{0}+B_{1} T+\cdots,
$$

AMS (MOS) subject classifications (1970). Primary 14E25.

Key words and phrases. Embedding, obstruction, Chern-classes.

${ }^{1}$ Research supported in part by NSF under grant GP29026X, and by the Norwegian Research Council for Science and the Humanities. 
of which we only need $B_{0}, B_{1}, \cdots, B_{n}$. In fact, we put

$$
\begin{aligned}
& \beta_{j}=\sum_{i=0}^{j-n}(-1)^{i}\left(\begin{array}{c}
n-i \\
j-i-n
\end{array}\right)\left(B_{i}-d^{2}\left(\begin{array}{c}
n+1 \\
i
\end{array}\right)\right), \quad n \leqq j \leqq 2 n,{ }^{2} \\
& \beta_{j}=1 \text { for } j<n, \quad \beta_{j}=0 \text { for } j>2 n .
\end{aligned}
$$

Definition. For all integers $m$ the sequence $\left(\beta_{m}, \beta_{m+1}, \cdots\right)$ is called the mth embedding obstruction of the embedded variety $(X, i)$.

In [2] the following result is proved:

THEOREM. If $m<N$, then $X$ can be embedded into $\boldsymbol{P}_{k}^{m}$ via a projection from $\boldsymbol{P}_{k}^{N}$ if and only if the mth obstruction vanishes, i.e.

$$
\left(\beta_{m}, \beta_{m+1}, \cdots\right)=(0,0, \cdots) \text {. }
$$

This implies at once the well-known and classical (see E. Lluis [5]):

Corollary. $m(X, i) \leqq 2 n+1$.

For $n=1$ and $m=2$ we obtain the well-known genus-formula

$$
g(X)=\frac{1}{2}(d-1)(d-2)
$$

which is necessary and sufficient for when the nonsingular curve $X$ can be projected isomorphically onto a plane curve. For $n=2, m=3$, we get that a nonsingular surface $X$ in $\boldsymbol{P}_{k}^{N}$ can be embedded into $\boldsymbol{P}_{k}^{3}$ via a projection if and only if

$$
\begin{aligned}
\operatorname{deg}\left(K_{X}\right) & =(d-4) d \\
\left(K_{X}^{2}\right) & =(d-4)^{2} d \\
p_{a}(X) & =\frac{1}{6}(d-1)(d-2)(d-3)
\end{aligned}
$$

Again $d=\operatorname{deg}(X), K_{X}$ is the canonical divisor and $p_{a}(X)$ the arithmetic genus of $X$. The necessity of (3) was noted by Iversen [4].

It should be easy to compute formulas similar to (2) and (3) in any dimension $n$ by means of (1), and thus obtain a characterization (in terms of classical invariants like $\left.K_{X}, p_{a}(X)\right)$ of those nonsingular varieties $X$ in $\boldsymbol{P}_{k}^{N}$ which can be projected isomorphically onto a hypersurface in $\boldsymbol{P}_{k}^{n+1}$. Of course (1) with $m=n+1$ gives such a characterization, in terms of the degrees of certain monomials in the Chern-classes of $X$.

Another application of the theorem is to Abelian varieties. In fact, the question of embeddings for Abelian varieties is resolved as follows: Let $X_{\hookrightarrow} \boldsymbol{P}_{k}^{N}$ be an $n$-dimensional Abelian variety. Then:

(i) $X$ can always be embedded into $\boldsymbol{P}_{k}^{2 n+1}$ via a projection from $\boldsymbol{P}_{k}^{N}$;

(ii) $X$ can be embedded into $\boldsymbol{P}_{k}^{2 n}$ via a projection from $\boldsymbol{P}_{k}^{N} \Leftrightarrow \operatorname{deg}(X)=$ $\frac{1}{2}\left(\begin{array}{c}2 n+1 \\ n\end{array}\right)$;

(iii) $X$ cannot be embedded into $\boldsymbol{P}_{k}^{2 n-1}$.

${ }^{2}$ AdDED IN PROOF. Using standard combinatorial identities, one easily checks that $(-1)^{j-n} \beta_{j}=\left(\sum_{i=0}^{j-n}\left(\begin{array}{c}j+1 \\ j-n-1\end{array}\right) d_{\imath}\right)-d_{0}^{2}$. 
For $n=1$, (ii) gives $\operatorname{deg}(X)=3$ which is no surprise, and for $n=2$ we get $\operatorname{deg}(X)=10$. The necessity of this condition for the embedding of a 2-dimensional Abelian variety into $\boldsymbol{P}_{k}^{4}$ was noted by Horrocks and Mumford in [3, Theorems 5.1 and 5.2].

It should be noted that [2] deals only with embedded projective varieties. For a given projective variety $X$, one may ask for the least integer $e=e(X)$ such that $X$ may be embedded into $\boldsymbol{P}_{k}^{e}$. If $X$ is given as a subvariety of some $\boldsymbol{P}_{k}^{N}$, one may very well have $m(X)>e(X)$. Nevertheless, calculation of $m(X)$ can be used to obtain upper and lower bounds for $e(X)$, see for example the computation for Abelian varieties referred to above. In order to compute $e(X)$, one must find the projective embeddings $i$ of $X$ for which $m(X, i)$ is minimal, i.e., for which the embedding obstruction is as nice as possible. We hope to return to this question later.

\section{REFERENCES}

1. M. F. Atiyah, Immersions and embeddings of manifolds, Topology 1 (1962), 125-132. MR 26 \#3080.

2. A. Holme, Embedding-obstruction for algebraic varieties. I. (to appear).

3. G. Horrocks and D. Mumford, A rank 2 vector bundle on $P^{4}$ with 15.000 symmetries, Topology 12 (1973), 63-68.

4. B. Iversen, Numerical invariants and multiple planes, Amer. J. Math. 92 (1970), 968-996. MR 45 \#5135.

5. E. Lluis Riera, Sur l'immersion des variétés algébriques, Ann. of Math. (2) 62 (1955), 120-127. MR 17, 87.

Department of Mathematics, University of Bergen, Bergen, Norway 